\title{
L'élite politique sous Gorbachev et Eltsine au debut de la période de transition : étude analytique de réputation
}

\section{David Lane}

\section{(2) OpenEdition \\ Journals}

Édition électronique

URL : http://journals.openedition.org/conflits/333

DOI : 10.4000/conflits.333

ISSN : 1777-5345

Éditeur :

CCLS - Centre d'études sur les conflits lilberté et sécurité, L'Harmattan

Édition imprimée

Date de publication : 15 mai 1995

ISSN : 1157-996X

Référence électronique

David Lane, «L'élite politique sous Gorbachev et Eltsine au debut de la période de transition : étude analytique de réputation », Cultures \& Conflits [En ligne], 17 | printemps 1995, mis en ligne le 15 mars 2006, consulté le 30 mars 2021. URL : http://journals.openedition.org/conflits/333 ; DOI : https:// doi.org/10.4000/conflits.333

Ce document a été généré automatiquement le 30 mars 2021.

Creative Commons License 


\title{
L'élite politique sous Gorbachev et Eltsine au debut de la période de transition : étude analytique de réputation
}

\author{
David Lane
}

Il y a quatre manières de procéder à l'analyse du leadership politique ${ }^{1}$ : l'étude des positions, l'étude de la réputation, l'étude de la décision, ou encore, celle de la répartition ${ }^{2}$. Bien que des approches décisionnelle et réputationnelle aient pu être utilisées pour les biographies des leaders, comme Khrouchev, l'analyse des positions a longtemps dominé les travaux occidentaux consacrés à la politique soviétique, principalement parce c'était la plus facile à mettre en oeuvre. Cependant, durant les périodes d'instabilité politique et de transformations politiques rapides, l'étude des positions peut s'avérer d'une moindre utilité: on peut assister en effet à des changements d'acteurs et des institutions politiques. Alors que l'opacité des processus de décision en Union Soviétique posait des problèmes importants aux chercheurs, la chute de l'ordre ancien a rendu difficile la définition du leadership dans une société en transition. Le pouvoir politique ne présente peut-être pas une configuration uniforme, et les personnes occupant des positions politiques définies peuvent manquer d'autorité. Donc, l'approche "positionnelle", qui prédominait dans les études sur l'Union Soviétique, est devenue aujourd'hui moins pertinente. Des constellations et coalitions changeantes d'individus, de groupes et d'institutions peuvent être repérées grâce à la perspicacité de personnes bien informées, par la méthode réputationnelle. Le politiste peut ne pas avoir d'autre alternative que d'adopter la démarche de l'anthropologue, à savoir demander aux membres de la tribu : "qui sont vos chefs ?" L'avantage de cette technique, si on l'utilise correctement, réside dans le fait qu'elle tient compte du point de vue de ceux qui sont impliqués dans les jeux politiques et qui connaissent les individus influents. 
Cette méthode peut présenter néanmoins des inconvénients et devrait utiliser avec précaution. En effet, une évaluation subjective de celui qui a de l'influence peut refléter les préférences des personnes interrogées, et peut être fondée sur une simple rumeur ou ne reposer sur aucune preuve réelle. En effet, la méthode réputationnelle révèle les différences de perception entre individus différents sur la puissance : toutes ne peuvent être justes et sans ambiguité. Mais, en l'absence de données et d'observations sur le processus de prise de décision, cette méthode peut souvent donner des indications sur les groupes d'individus constituant l'élite politique - même si leur influence réelle reste indéterminée. En outre, les individus et les intérêts mis en évidence par de telles enquêtes peuvent constituer une base pour des recherches plus poussées et une source d'hypothèses intéressante. Enfin, cette méthode peut révéler une partie importante de l'opinion public informée: par exemple, quelle personne est censée être un agent politique, ou quelles institutions et forces sociales sont supposées jouer un rôle politique important. De telles différences de perception de l'élite dominante, et de son évolution constituent également un élément important de la culture politique.

La méthode réputationnelle peut s'avérer particulièrement appropriée pour l'analyse des sociétés où des institutions politiques et économiques sont centralisées et où le leadership politique s'appuie sur, relativement, peu de réseaux politiques - ce qui était probablement le cas de la Russie sous Mikhail Gorbachev et Boris Eltsine en 1991 et $1992^{3}$.

Cette analyse repose donc sur une étude réputationnelle basée sur l'opinion d'individus pouvant être considérés comme des experts de la politique de l'URSS sous Gorbachev et de la Russie de Boris Eltsine. Quarante-huit personnes, issues de trois structures différentes, ont été interviewées à l'automne 1992. La composition de l'échantillon était la suivante : des députés importants du Soviet Suprême ou du Parlement russe $(\mathrm{N}=16)$, des académiciens ou conseillers $(\mathrm{N}=16)$; des membres du gouvernement $(\mathrm{N}=16)$. Tous les interlocuteurs ont été interviewés à Moscou, par des Russes.

Ils ont été sélectionnés d'égale manière parmi des personnes qui disposaient d'une connaissance et avaient l'expérience de la période de Gorbachev, ainsi que de celle d'Eltsine. Les interviews ont été réalisés par téléphone par des membres de l'Institut de sociologie de l'Académie russe de sciences ${ }^{4}$. Les questions étaient combinées de façon à déceler les groupes influents au plus haut niveau sous Gorbachev (à partir du moment où il est devenu Secrétaire Général du Parti jusqu'au coup d'Etat d'août 1991) et sous Eltsine (à partir du coup d'Etat jusqu'à la mi-1992). Cette étude visait également à déterminer les institutions et les forces socio-politiques qui avaient contribué à l'élaboration de la politique du pays durant ces périodes.

Quatorze questions au total, regroupées en groupes de deux questions, ont été posées, trois groupes relatifs à la période de Gorbachev et quatre à celle d'Eltsine. Pour le premier, on a défini trois sujets, ayant chacun deux parties : la première était liée aux personnes auxquelles nos interlocuteurs attribuaient de l'influence, et la seconde aux institutions ou forces qu'ils pensaient avoir été puissantes. Dans le but de mettre en évidence différents éléments de l'élite politique, nous avons posé des questions concernant des niveaux différents dans la formation des décisions: la politique intérieure, la politique économique ainsi que les relations avec d'autres Etats. Nous avons posé des questions sur la sécurité intérieure - le Traité de l'Union (Soyuzny dogovor) (questions 1 et 2), sur l'économie - l'introduction des coopératives, et des entreprises privées ( 3 et 4 ) et sur les relations internationales; le retrait de l'armée 
rouge de l'Europe de l'Est (5 et 6). Pour la période d'Eltsine, on a utilisé une stratégie similaire en ce qui concerne la politique intérieure, sur l'interdiction du Parti communiste (9 et 10); sur l'introduction de l'économie de marché (13 et 14) et sur la chute de l'URSS (11 et 12). Nous avons également demandé quelles étaient selon nos interlocuteurs, les personnes les plus puissantes au moment où les interviews eurent lieu - en octobre et novembre 1992. A chacune des questions, on a demandé cinq réponses. Les réponses n'étaient pas limitées et on n'a suggéré aucune personne ni groupe, ni institution. (On a pourtant demandé aux interlocuteurs de ne pas mentionner Gorbachev ou Eltsine dans les questions les concernant).

Les personnalités influentes sous Gorbachev

Qui était donc considéré comme ayant de l'influence sous Gorbachev ? Le tableau 1 (cidessous) montre la diversité des personnes citées par tous les interlocuteurs (48 en total) comme ayant eu de l'influence dans trois domaines importants. Comme les questions étaient ouvertes, un grand nombre de personnes a été cité, 66 en tout, dont seize sont mentionnés dans le tableau (la plupart des autres ne sont guère connus des lecteurs occidentaux). Des membres de l'élite politique ont été cités, mais également les personnes cherchant à l'influencer de l'extérieur l'Union Soviétique (comme des chefs d'Etat étrangers).

Tableau 1 : Composition et structure des élites politiques sous Gorbachev

Question 1 Question 3 Question 5 Total

Iakovlev 19183572

Shevarnadze 1153450

Nazarbaev 290029

Yel'tsine 230023

Abalkine 016117

Kravchuk 140014

Ryzhkov 013013

Shakhnazarov 72413

Question 1 Question 3 Question 5 Total

Kohl 001212

Bush 101011

Agabegian 0909

Shatalin 2608

Bakatin* 2147

Cherniaev* 2237

Reagan* 0077

Thatcher* 1067

Autres* 444622114

En examinant la fréquence des noms, on trouve quelques indications concernant les influences politiques. Il est bien évidemment, fait allusion à des personnes différentes selon les périodes, durant le temps où Gorbachev était le chef de l'Etat. Les réponses à la question 1 (sur le Traité de l'union) ${ }^{5}$, montrent que dans le domaine des relations entre les différentes républiques, on pensait déjà que Gorbachev était sous l'influence 
de personnes n'appartenant pas à l'élite au pouvoir qu'il avait lui même constituée et choisit : les chefs des républiques Nazarbaev, Eltsine et Kravchuk étant trois des quatre personnes ayant le plus d'influence. Les réponses à la question 3 (sur le commerce privé) ${ }^{6}$ confirment que Alexander Iakovlev (ancien ambassadeur de l'Union soviétique au Canada entre 1973 et 1983, et ensuite, à partir de 1987, membre permanent du Politbureau et associé intime de Gorbachev) a été l'homme qui a le plus d'influencé Gorbachev. En étudiant ces réponses, on remarque une configuration différente de celle obtenue pour le Traité de l'Union. Dans le domaine économique, les économistes Leonid Abalkin (ancien chef de l'Institut d'économie à l'Académie des Sciences Sociales), Nikolai Ryzhkov (qui travaillait au Gosplan jusqu'en 1982, lorsqu'il est devenu chef du Département de l'économie au Comité Central, et en 1985 chef du Conseil des Ministères de l'Union Soviétique), Abel Aaenbegian (professeur d'économie et membre de l'Académie des Sciences de l'URSS, également directeur de l'Institut pour les forces de production et les ressources naturelles) et Stanislav Shatalin (ancien chef du département économique à l'Université de Moscou et membre de l'Académie des Sciences de l'URSS) étaient supposés avoir joué un rôle beaucoup plus important, tandis que les politiques étaient prédominants dans la discussion sur le Traité de l'Union. Concernant la question 5 (sur la décision de retrait des forces armées de l'Europe de l'Est) ${ }^{7}$, un autre schéma se dessine encore. Suivant Alexander Iakovlev et Eduard Shevardnadze - membres importants du Politburo - il y a quatre hommes politiques étrangers : Le Chancelier Helmut Kohl, les Présidents des Etats-Unis, Georges Bush et Ronald Reagan et le Premier Ministre britannique Margaret Thatcher. Leur "score " cumulé est de 35, comme Iakovlev. Le chef du KGB, Vadim Bakatin, n'a été cité que 5 fois. Notre compréhension de la politique doit prendre en compte sans aucun doute les intérêts internationaux ainsi que les pressions exercées par l'étranger. En prenant en compte ces trois groupes d'évaluation, on peut former une liste des personnes influentes sous Gorbachev, formant l'élite politique (comme on peut le voir dans la colonne 4). Les hommes les plus importants (ayant 9 mentions ou plus) figurent dans le graphique 1 , où on peut aussi noter le domaine dans lequel ils ont de l'influence.

On remarque la prédominance des leaders communistes: Alexander Iakovlev et Edouard Shevardnadze (membre du Politbureau depuis 1978 - membre titulaire depuis 1985 - et Ministre de Affaires Etrangères); des hommes bénéficiant d'une assise régionale - Leonid Kravchuk, le Président de l'Ukraine, et Nursultan Nazarbaev, le Président du Kazakhstan - tous les deux étant, bien sûr, les leaders communistes les plus puissants dans leurs républiques. Les chefs d'Etats étrangers, Helmut Kohl, le chancelier de la RFA, et Georges Bush, le Président des Etats-Unis, ainsi que les conseillers Leonid Abalkin et Georgii Shakhnazarov (qui a travaillé comme fonctionnaire dans l'appareil du Comité central, était membre de l'Académie des Sciences et a été le premier vice-président de l'Association Internationale des Sciences Politiques), Abel Aganbegian et Anatolii Cherniaev (ancien Vice-président du département international du Comité central du Parti entre 1970 et 1986, avant qu'il ne devienne le conseiller en politique étrangère de Gorbachev) sont aussi présents. On comparera plus tard les structures politiques auxquelles appartiennent les personnes citées ici avec celles citées pour Eltsine

Les institutions et les forces politiques influentes sous Gorbachev

L'un des mystères de la chute de l'Union Soviétique réside dans la question de savoir quelle institution a contribuée à la politique qui a fait éclater le régime et tomber le Parti communiste. Quelques questions complémentaires sur les institutions visaient à 
éclaicir ce point. Les réponses sont rassemblées dans le tableau 2 (voir ci-dessous). La question $\mathrm{n}^{\circ} 2$ portait sur les forces socio-politiques ayant une certaine influence dans la formation du Traité de l'union ${ }^{8}$, la question $n^{\circ} 4$ concernait les forces socioéconomiques qui ont soutenu Gorbachev dans la formation des coopératives ${ }^{9}$, et la question $n^{\circ} 6$, portait sur les forces socio-politiques qui avaient influé sur la décision de retirer les forces armées de l'Europe de l'Est ${ }^{10}$. Les totaux figurent dans la dernière colonne.

Tableau 2:

Question 2 Question 4 Question 6 Total

PCUS 2212640

Forces externes 732838

Mouvements nationalistes (républiques et régions) 270532

Intelligentsia 4141331

Démocrates 861428

Nomenklatura 87520

Mafia 115016

Bourgeoisie 311115

milieu judiciaire 211013

Opinion publique 29213

Gouvernement (ministères du secteur économique) 110011

Armée 60410

Medias 16310

Russie Démocratique 5229

Leaders des républiques et des régions 9009

Bureaucratie centrale 1708

KGB 5117

Soviet Suprême 7007

Complexe militaro-industriel 5005

Leaders de haut-rang 3014

Administration (Russie) 3003

Députés 3003

Diplomates 0033

Cercle Eltsine 30003

Cercle Gorbachev 1023

Gouvernement 1023

Komsomol 0303

Bureaucraties des régions 2002

Les leaders centraux des Partis 2002

Libéraux 0022

Opposition 1102

Soviets Suprêmes (Républiques) 2002 
Agrairiens 0101

Le Parti démocratique de Russie 1001

Partisans de Iakovlev 1001

Mouvement pour une Réforme Rémocratique 1001

Néo-communistes 1001

Mouvement paysan 0101

Partisan du parti de la Russie Libre 10001

Syndicats 0101

Travailleurs collectifs 0101

Jeunesse 01101

Dans les réponses à la question $\mathrm{n}^{\circ} 2$, on peut isoler les groupes influents qui n'appartenaient pas à l'élite gorbachévienne, définie par les questions précédentes portant sur les individus (cf. tableau 1 et graphique 1). Des personnalités des mouvements nationalistes dans les républiques (27) ont souvent été plus citées que le Parti communiste de l'Union Soviétique (22). Dans ce tableau, les démocrates sont rarement mentionnés (8). Ces résultats indiqueraient donc que Gorbachev a été poussé à agir par les forces nationalistes dans le cadre du Traité de l'Union.

Pour les questions relatives à l'introduction de l'entreprise privée, on remarque la prédominance des forces économiques n'appartenant à l'establishment du pouvoir politique. La «mafia» (terme souvent employé pour désigner aussi bien des commerçants quels qu'ils soient, que des hommes d'affaires en général, ou des spéculateurs) et la «bourgeoisie » totalisent un score très important par rapport au groupe le plus proche (l'intelligentsia). Cette dernière catégorie comprend également des professionnels, comme des médecins, capables de vendre leurs services sur le marché. Il semble que les coopératives aient été favorisées par de nombreuses institutions, comme les sections du Parti communiste, ou encore les fonctionnaires gouvernementaux du secteur de l'économie (et également la bureaucratie centrale et la nomenklatura).

Enfin, pour ce qui concerne les questions relevant des relations internationales, les forces externes, ce qui peut paraître surprenant, ont de loin été le plus rapportées, suivies par des forces sociales générales (des démocrates et des intellectuels). Le PCUS et la nomenklatura ne se voient attribuer que peu d'influence dans ce domaine. Contrairement aux forces sociales générales, il a été attribué peu de poids aux institutions internes dans la détermination de la politique étrangère. Cette dernière semble être définie en grande partie par l'élite politique proche de Gorbachev.

Avant d'analyser plus en détail ces résultats, nous allons nous intéresser aux réponses apportées à ces mêmes questions mais cette fois après l'arrivée au pouvoir d'Eltsine. Nous pourrons allors comparer les variations dans la composition des élites politiques au cours des deux périodes.

Les personnalités influentes sous Eltsine

Tableau 3

Question 9 Question 11 Question 13 Question 7 Total

Burbulis 34221234102

Gaidar 04333067

Cultures \& Conflits, 17 | printemps 1995 
Kravchuk 1390040

Shakhrai 23100235

Khasbulatov 4202834

Rutskoi 3042431

Vol'skii 0042024

Poltoranin 1052522

Popov 1124320

Shushkevich 0200020

Chubais 0017219

Skokov 0011617

Shokhin 0012214

Petrov 0011213

Gorbachev* 04408

Nazarbaev 0100010

Autres* 48496928193

* non reporté sur le graphique 2

Pour étudier les aspects du leadership sous Eltsine, nous avons procéder de la même façon. Le tableau 3 (ci-dessus) montre les résultats obtenus. La première question concernait l'interdiction du PCUS ${ }^{11}$. La répartition des réponses figure dans la première colonne (Q. 9). La fréquence des réponses indique qu'Eltsine a été influencé de façon considérable par ses associés politiques proches: Gennadii Burbulis qui était le secrétaire d'Etat attaché au Président de la Fédération de Russie jusqu'au 26 novembre 1992, lorsqu'il est devenu le chef du groupe des conseillers du Président; Sergei Shakhrai, vice-président $\mathrm{du}$ gouvernement responsable des questions juridiques jusqu'au 5 novembre 1992, puis vice-président du gouvernement responsable des questions de nationalité et chef du comité d'Etat pour la politique interethnique; Gavriil Popov, le chef de la branche russe du mouvement pour les réformes démocratiques et maire de Moscou jusqu'en juin 1992, et Mikhail Poltoranin, qui était le ministre de la presse et de l'information et vice-président de la Fédération de Russie nommé en novembre 1992 à la tête du Centre Fédéral de l'information. La deuxième question portait sur la dissolution de $\mathrm{l}^{\prime} \mathrm{URSS}^{12}$. On remarque des influences extérieures importantes à la Russie, notamment celles de Leonid Kravchuk (Président de l'Ukraine), Nursultan Nazarbaev (Président du Kazakhstan) et Stanislas Shushkevich (Président de la Biélorussie). Cela suggère que le pouvoir politique a suivi des élites qui n'appartenaient pas à la Fédération de Russie. Il semble donc qu'on ne puisse attribuer la décision de dissoudre l'URSS à l'entourage politique d'Eltsine.

Pour déterminer les influences sur les décisions économiques, nous avons posé une question relative à l'introduction de l'économie de marché ${ }^{13}$. Le tableau 3 , question 13 , montre la répartition des réponses. Outre, Gennadii Burbulis, déjà mentionné, les quatre personnalités le plus citées ont été Egor Gaidar, le premier vice-premier ministre du gouvernement de la Fédération de Russie et, pendant la période des interviews, le Premier Ministre russe, Anatolii Chubais, ancien conseiller et viceprésident du Soviet de Leningrad, puis le vice-président du gouvernement responsable des privatisations, et Alexander Shokhin, le vice-président du gouvernement de la Fédération de Russie responsable des affaires sociales et des relations économiques 
avec l'étranger. Tous les trois sont des économistes, ce qui indique que l'intelligentsia économique a joué un rôle important dans ce domaine.

Ces questions concernent des sujets importants, mais elles ne couvrent pas tous les domaines du débat politique, et les réponses pourraient exclure des gens ayant de l'influence dans d'autres domaines et ayant un potentiel d'action politique. On a donc posé une question générale sur les personnes auxquelles nos interlocuteurs attribuaient le plus d'influence ${ }^{14}$ (Voir les réponses dans la cinquième colonne, Question $\mathrm{n}^{\circ} 7$ ). Nous discuterons ci-dessous des antécédents institutionnels de ces personnalités. Il est pourtant important de noter, que des six personnes les plus fréquemment citées, Gennadii Burbulis, Alexander Rutskoi, le Vice-président de la Russie, ainsi que le chef du Parti du peuple pour la Russie libre et un des chefs de l'Union civique, Egor Gaidar et Iurii Skokov - le secrétaire du Conseil de sécurité de l'époque - étaient tous membres du gouvernement de la Russie ou du pouvoir exécutif. Seuls Ruslan Khasbulatov, Président du Presidium du Soviet Suprême de la Fédération de Russie (chef du Conseil de l'Assemblée inter-parlementaire de la Communauté des Etats Indépendants depuis septembre 1992) et Arkadii Volskii, Président de l'Union russe des industriels et entrepreneurs, un des chef de obnovleinie (l'Union russe du renouvellement) et un des chefs de l'Union civique, représentaient un contrepoids au pouvoir d'Eltsine. Enfin, pour mettre en évidence la composition de l'élite politique présente autour d'Eltsine, nous avons additionné les réponses aux quatre questions (cf. colonne total du tableau 4 ci-dessous).

Sur le graphique 2 ci-dessus, on a indiqué les domaines dans lesquels on attribue de l'influence à ces personnes. Parmi les leaders les plus influents, on peut distinguer trois groupes - et donc une élite politique segmentée. Il y a d'abord les membres du gouvernement et les conseillers du Président (Burbulis, Gaidar, Rutskoi, Poltoranin, Shakhrai, Chubais et Popov), ensuite, les chefs des autres républiques (Kravchuk et Shushkevich) et enfin les personnes n'appartenant pas au pouvoir exécutif Khasbulatov et Volskii - avec Rutskoi appartenant à la fois à ce groupe et au premier. Dans le tableau 3, un grand nombre de personnes n'ont été que rarement mentionnées, ce qui indique une fragmentation de l'élite, ou l'importance du nombre des personnes ayant des relations avec les principaux leaders. C'est également ce qui ressort, et qui frappe, du graphique 2, à savoir la fragmentation de l'élite politique. Seul Burbulis a une influence significative dans tous les secteurs. On voit nettement qu'après la formation des Etats indépendants, qui a suivi de peu la prise de pouvoir d'Eltsine en août 1991, Kravchuk, Shushkevich et Nazarbaev ont perdu de leur influence en Russie. Rutskoi et Volskii, en revanche, sont désormais considérés comme membres de fractions de l'élite d'où émergeaient des contre-élites qui défiaient le Président.

En comparaison avec l'élite qui émerge de l'analyse de réputation appliquée à l'époque gorbachevienne, on peut constater de nombreux changements. L'élite communiste a disparu : ceux qui ont été cités l'ont été notamment par référence aux anciens membres de l'élite (comme celle de Gorbachev) dont l'activité a été perçue comme ayant précipité les actions d'Eltsine. A l'époque de Eltsine, les membres du Gouvernement étaient nettement prédominants, suivis par des leaders élus. Les chefs de partis et de groupes jouaient un rôle mineur, et les chefs d'Etat étrangers, les industriels, les entrepreneurs ainsi que les militaires ont eu très peu d'influence.

Les forces socio-politiques soutenant Eltsine 
Comme on l'a déjà fait remarquer, une étude de l'origine des leaders ne peut pas prendre en compte les changements d'allégeance des individus, et souvent les leaders n'agissent pas par rapport au groupe à la tête duquel ils se trouvent ou qu'ils sont sensés représenter. On a donc posé à nos interlocuteurs une série de questions complémentaires sur les forces qui selon eux influençaient les mêmes options politiques. Nos interlocuteurs ont estimé qu'il était plus difficile de répondre à ces questions car elles demandent une connaissance plus abstraite de la politique.

Tableau 4

Q.10 Q.12 Q.14 Q.8 Total

Bourgeoisie 4532849

Russie Démocratique 156101546

Démocrates 18910744

Mouvements nationalistes (républiques et régions) 1330741

Union civique 0032326

Gouvernement (ministères du secteur économique) 0071421

Nomenklatura 664420

Patriotes nationaux 2311420

Intelligentsia 4310118

Medias 432615

Forces externes 166114

PCUS 630413

Q.10 Q.12 Q.14 Q.8 Total

Mafia 315413

Opinion publique 326213

Députés 211812

Cercle Eltsine 430512

Administration (Russie) 225211

Leaders des républiques et des régions 04059

Opposition 01067

Agrairiens 00437

Bureaucratie centrale 13116

Complexe militaro-industriel 00066

Comité d'Etat de l'état d'urgence 600006

Parti de la liberté économique 00516

Armée 00055

Gouvernement 00235

Syndicats 00145

Parti du peuple de la Russie libre 00415

Partis dans les républiques 00415

Assemblée du peuple russe 00055

Soviet Suprême 00224 
Le Parti démocratique de Russie 00314

Néo-communistes 20024

Lobby du monde des affaires 10214

Milieux judiciaires 120003

Les leaders centraux des Partis 00123

Mouvements des travailleurs 20013

Travailleurs collectifs 000033

Partis 20002

Mouvement pour une Réforme Rémocratique 00022

Mouvement paysan 00022

Hauts fonctionnaires 00202

KGB 100001

Leaders de haut-rang 01001

Clergé 100001

Q. 10 Q. 12 Q. 14 Q. 8 Total

Komsomol 00101

Bureaucraties des régions 01001

Libéraux 000011

Mouvement chrétien démocrate russe 00101

Anticommunistes 10001

Partisans de Gaidar 00101

Parti socialiste 00011

Jeunesse 00101

On a d'abord demandé quelles étaient les forces socio-politiques qui avaient eu une influence décisive sur l'interdiction du Parti communiste ${ }^{15}$. Les réponses figurent dans la première colonne du tableau 4 , les principales forces socio-politiques citées ici étant l'opposition démocratique sous Gorbachev. Plusieurs interlocuteurs ont considéré que le Parti lui-même, et son élite, ont le plus oeuvré pour leur propre chute (quelques uns ont répondu que c'était le Comité d'Etat pour l'état d'urgence et le PCUS). Dans ce tableau, il y a peu de références aux patriotes nationaux ou aux forces dans les républiques et les régions.

En second lieu, on a demandé quelles étaient les forces socio-politiques ayant joué un rôle dans l'éclatement de l'URSS et la formation de plusieurs Etats indépendants ${ }^{16}$. Les réponses qui figurent dans la deuxième colonne du tableau 4 montrent des forces très différentes de celles responsables de l'interdiction du PCUS, en particulier le rôle joué par des mouvements dans les républiques et les régions, soutenus par «les forces démocratiques ».

La troisième question portait sur les forces socio-politiques ayant soutenu la prise en compte des forces du marché17, on voit encore une nouvelle distribution dans la troisième colonne du tableau 4 . La "bourgeoisie" était nettement au premier rang dans l'esprit de nos interlocuteurs, suivie par des forces démocratiques ainsi que par l'intelligentsia. 
Enfin, pour les raisons évoquées précédemment, on a posé une question générale sur les forces socio-politiques les plus puissantes en Russie en octobre-novembre $1992^{18}$. La liste figurant dans la troisième colonne du tableau 4, montre une répartition encore différente. On remarque que la dispersion des réponses est bien plus importante que précédemment. Au sommet, il y a trois différentes groupes : «l'Union civique » (23) et les patriotes nationaux (14), "la Russie démocratique» (15) et les fonctionnaires gouvernementaux du secteur de l'économie (14). La "Russie démocratique ", principal soutien social et politique d'Eltsine lors de son ascension au pouvoir ne vient pas en tête, et on peut remarquer que les élites politiques sont composés d'acteurs très différents, comme on le verra par la suite.

Plus surprenant peut-être, le plus grand groupe qui semble avoir le plus d'influence sous Eltsine est la «bourgeoisie " (à laquelle on devrait ajouter la «mafia ») suivi par son groupe politique: la «Russie démocratique». Le mouvement nationaliste a beaucoup plus d'importance que sous Gorbachev. Les institutions et les forces de la Russie soviétique ayant subi un sévère déclin - le PCUS, le KGB et l'armée ne sont mentionnés que rarement. Il est aussi intéressant de constater que des groupes sociaux, comme les paysans, les ouvriers et l'Eglise, sont rarement cités.

<!--SPIP--> Comparaison entre les élites soutenant Gorbachev et Eltsine

Dans l'analyse précédente on s'est concentré sur les réponses données par les personnes interrogées, mais on a utilisé ces informations pour les appliquer aux groupes politiques soutenant l'élite politique. En comparant le soutien de l'élite sous Gorbachev et sous Eltsine, on a construit deux tableaux comparatifs. Le premier est fondé sur l'origine des personnes mentionnées dans les tableaux 1 et 3), l'autre sur les institutions (tableau 2 et 4 ).

Les biographies des personnes citées ci-dessus sont intéressantes pour les soviétologues et russologues, bien qu'il n'en existe pas pour toutes les personnes citées par nos interlocuteurs. En outre, on aurait besoin de plus amples informations sur les groupes politiques dont elles sont issues et qu'elles pourraient représenter. Dans l'analyse suivante, j'ai comparé les antécédents institutionnels des élites politiques sous Gorbachev et sous Eltsine. Chaque personne mentionnée dans les questions 1, 3 et 5, ainsi que 9, 11, 13 et 7 (voir tableaux 1 et 3) a été rattachée à un groupe spécifique comme on peut le voir dans tableau 5 . Avec une scène politique aussi changeante et une telle mobilité politique, on ne peut affilier sans risque une personne à un groupe. Ici, on a choisi d'associer la personne à la position ayant le plus de prestige, ou pour laquelle la personne est le plus connue. Cette évaluation sera complétée lorsqu'on prendra en compte l'identification des « forces socio-politiques » par nos interlocuteurs.

On a utilisé les douze catégories suivantes : Les dirigeants du Parti communiste (CPE), les personnes ayant occupé une position importante sous Gorbachev et qui n'ont pas quitté le Parti ; l'élite politique élue (ELE) - des gens ayant des positions importantes sous Eltsine et qui ont été élus (comme maires ou Présidents) ; l'élite du Gouvernement (GEM) - membres du gouvernement; les chefs d'Etat étrangers (FL) (chefs d'Etats n'appartenant pas à l'ancienne Union soviétique); les conseillers (ADV); les universitaires ayant des intérêts politiques (AV); les chefs des partis ou groupes politiques (PAL); les fonctionnaires du Parti communiste (CPF) (les apparatchiks de niveau inférieur); les représentants élus (ELR) (députés du Soviet Suprême); les personnes apparentées aux leaders $(\mathrm{K})$; les écrivains et les pontifs $(\mathrm{W})$; les leaders des républiques (RL) - qui ne sont pas élus Présidents; les industriels et les entrepreneurs 
(ICE); les dirigeants des médias (MC) (la presse, la télévision, la radio); les chefs militaires (M) (ou anciens chefs); et une catégorie "résiduelle» pour les autres (Others). (Voir le graphique 3).

Il s'agissait ici de rattacher chaque personne à un groupe : on définit les leaders du Parti communiste sont définit par les interviewés comme ayant une position dominante au sein du parti (membres du Politbureau), l'élite politique élue est composée d'individus élus au poste de président (comme Karimov Président de l'Ousbekistan), les députés (comme Tat'iana Koriagina) étant placés dans la catégorie des représentants élus. Un grand nombre de personnes citées cumule plusieurs rôles (par exemple député au parlement et conseiller). Dans ce cas, on a pris en considération leur statut politique le plus élevé. Ainsi, Nazarbaev est considéré comme appartenant à la catégorie de l'élite politique élue, plutôt qu'à celle des dirigeants du Parti. L'avantage de ce tableau est qu'il indique la distribution politique de toute l'élite "réputationelle», et non point uniquement de ceux qui sont bien connus. Il montre aussi les institutions et les forces sur lesquelles s'appuyaient les leaders « réputationnels » qui avaient de l'influence à la fin de la présidence Gorbachev, et comment elles ont changé sous Eltsine. Ce tableau donne des indications sur la structure politique de l'ensemble de l'élite politique.

Tableau 5 :

Code Gorbachev Eltsine

CPE Dirigeants du PC 10417

ELE Elite politique élue 77177

GEM Elite du Gouvernement 72257

FL Chefs d'Etat étrangers 402

ADV Conseillers 3780

AC Universitaires 178

PAL Chefs des partis ou groupes politiques 873

CPF Fonctionnaires du PC 70

ELR Représentants élus (députés) 61

K Apparentés aux leaders 50

W Ecrivains et pontifs 50

RL Leaders des républiques 46

ICE Industriels, entrepreneurs 28

MC Dirigeants des médias 21

M Militaire 10

On peut tirer plusieurs conclusions sur les structures des élites politiques sous Gorbachev et Eltsine. Elles ont sans nul doute changé de façon décisive. Considérons d'abord les individus qui ont influencé Gorbachev: ils sont issus de l'élite politique établie - principalement de la direction du Parti communiste puis des dirigeants du gouvernement et enfin des principaux dirigeants élus (comme les Présidents des Républiques). Ces groupes pourraient être considérés comme originaires d'une seule et même structure politique: presque tous ceux qui ont été le plus souvent cités occupaient des fonctions importantes au sein de l'élite communiste (Iakovlev, Shevardnadze, Nazarbaev, Kravchuk) et pourraient être définis comme des soutiens de Gorbachev. La seule exception est Eltsine, qui était l'un des opposants majeurs et 
représentatif de l'élite représentative élue. Ce qui étonne peut-être est le fait que les chefs d'Etat étrangers occupent une place importante, bien que cela soit dû à leur influence supposée sur la politique étrangère. Les conseillers, universitaires, journalistes et ainsi que ceux qui n'appartenaient pas à l'« ordre " politique n'étaient cités que plus rarement.

Sous Eltsine, l'élite communiste a disparu: le nombre peu élevé de réponses (17) s'explique en partie par les références à d'anciens membres de l'élite (Gorbachev en particulier) dont les activités antérieures ont influencé Eltsine, ou sont considérées comme à l'origine des actions des insurgés. L'élite du gouvernement est ici pleinement prédominante, suivie par les dirigeants élus et les conseillers. Des chefs de partis et de groupes sont représentés de façon significative. Les chefs d'Etat étrangers, les industriels et les entrepreneurs, ainsi que les militaires paraissent avoir joué un rôle d'une importance mineure. Une autre caractéristique de l'origine du leadership est l'absence de reférénce à des leaders associés avec un syndicat ou à des groupes de la classe ouvrière. Le nombre de chefs d'Etat étrangers chute considérablement, tandis que le nombre de chefs de partis et de groupes augmentent de façon significative.

Il est également intéressant de noter les personnes qui n'étaient pas citées par les interviewés. Les militaires, les représentants des ouvriers, les chefs des syndicats ainsi que les hommes d'affaires sont considérés comme ayant une influence négligeable. Dans les deux enquêtes, les femmes ne sont que rarement évoquées (seulement six femmes pour la période de Gorbachev - la première étant Mme Thatcher, suivi par Mme Gorbachev, et uniquement deux sous Eltsine), comme d'ailleurs, il convient de le noter, des membres des contre-élites des républiques (comme Vytautas Landsbergis, le Président de la Lithuanie). A l'exception d'Eltsine, importante certes, le programme de réformes lancé par Gorbachev était influencé par des élites en place ainsi que par dirigeants étrangers.

Le deuxième tableau comparatif concerne les forces sociales mentionnées ci-dessus. Ici, on tente d'élargir à différents groupes qui, d'une part ont soutenu Gorbachev dans son ascension et qui ont oeuvré pour sa chute et d'autre part, les groupes qui ont soutenu ou se sont opposées à Eltsine. Sur la base des réponses des tableaux 2 et 4 , on a réparti les institutions et les forces en six catégories :

1. L'appareil : les groupes administratifs, 2. Les groupes et les factions politiques, 3. Les forces économiques nouvelles, 4. Les forces régionales et éthniques, 5. Les groupes sociaux, et 6 . autres (une catégorie résiduelle).

Les totaux figurent dans le graphique 4, et les scores individuels dans le tableau 6 (ciaprès)

tableau 6 :

Code Gorbachev Eltsine

1 APPAREIL 17283

1 PCUS 620

1 Nomenklatura 2820

1 Armée 165

1 KGB 1221

1 Gouvernement (ministères du secteur économique) 121

1 Complexe militaro-industriel 106 
1 Bureaucratie centrale 96

1 Leaders de haut-rang 79

1 Administration (Russie) 69

1 Gouvernement 45

1 Komsomol 31

1 Diplomates 30

Code Gorbachev Eltsine

2 POLITICAL GROUPS/FRACTIONS 130197

2 Forces externes 4514

2 Forces Démocratiques 3644

2 Russie Démocratique 1446

2 Députés 612

2 Cercle Eltsine 612

2 Leaders centraux des partis 43

2 Cercle Gorbachev 40

2 Opposition 37

2 Parti du peuple de la Russie libre 25

2 Néo-communistes 24

2 Parti démocratique de Russie 24

2 Anticommunistes 24

2 Mouvement pour une réforme démocratique 22

2 Libéraux 21

2 Partisans de Iakolev 20

2 Union civique 026

2 Parti de la liberté économique 06

2 POLITICAL GROUPS/FRACTIONS 130197

2 Assemblée du peuple russe 05

2 Partis 02

Code Gorbachev Eltsine

2 Mouvement chrétien démocrate russe 01

2 Partisans de Gaidar 01

2 Parti socialiste 01

Gorbachev Eltsine

Code NOUVELLES FORCES ECONOMIQUES 3673

3 Bourgeoisie 1849

3 Mafia 1713

3 Agrairiens 17

3 Lobby du monde affaires 04

Gorbachev Eltsine

Code FORCES ETHNIQUES REGIONAL 8576 
4 Mouvement national dans les républiques 15941

4 Leaders des républiques et des régions 189

4 Bureaucracie des régions 41

4 Soviets suprêmes des républiques 40

4 Patriotes nationaux 020

4 Partis dans les républiques 05

Gorbachev Eltsine

Code SOCIAL 3828

5 Intelligentsia 3518

5 Mouvement Paysan 12

5 Travailleurs collectifs 13

5 Jeunesse 11

5 Clergé 01

5 Mouvement d'ouvriers 03

Gorbachev Eltsine

Code AUTRES 5653

6 Milieux judiciaires 153

6 Opinion publique 1513

Code AUTRES Gorbachev Eltsine

6 Soviet Suprême 144

6 Médias 119

6 Syndicats 15

6 PCUS 013

6 Comité d'Etat pour l'état d'urgence 06

graphique 4

Ici encore, il y a une différence statistique très significative entre les fréquences avec lesquelles les différentes structures sont mentionnées. Sous Gorbachev, l'appareil gouvernement et institutions bureaucratiques du Parti - est le groupe le plus important (172). A l'intérieur de la catégorie "groupes et factions politiques ", une contre-élite politique peut être distinguée, des forces extérieures (gouvernements occidentaux), suivis par les démocrates et « la Russie démocratique ». Des groupes de personnes (« Le clan d'Iakovlev ») commencent à apparaître, quoiqu'à un degré relativement faible, et que l'on peut considérer comme des ligues, préfigurant la formation d'un parti. Les forces nationales au sein des républiques et des régions occupent une place importante (85). En revanche, les forces économiques nouvelles sont peu mentionnées (36). Les groupes sociaux ne semblent pas importants (38), à l'exception de l'intelligentsia qui est citée de façon significative (35). Les travailleurs collectifs (1), les syndicats (1) et le mouvement paysan ont visiblement peu d'influence. L'opinion publique (15) et les médias(11) ne sont pas considérés comme cruciaux - ce qui peut paraître surprenant.

Sous Eltsine, la nouvelle élite a une autre origine. Cette analyse fait apparaître les six forces suivantes, les plus souvent citées : la bourgeoisie (49), la Russie démocratique (46), les forces démocratiques (44), les mouvements nationalistes dans les républiques et les régions (41), l'Union civique (26) et le secteur gouvernemental de l'économie (21). 
Le groupe le plus important est composé d'associations et factions politiques, avec la Russie démocratique, les démocrates et l'Union civique, suivis par une kyrielle de petits partis politiques. En outre, le mouvement nationaliste et les patriotes nationaux totalisent 61 références. L'autre évolution importante réside dans l'importance des forces économiques nouvelles : le lobby du monde des affaires, la mafia, 66 mentions en tout. Ceci est nettement supérieur aux institutions formant l'appareil: le secteur gouvernemental n'étant mentionné que 21 fois, et la nomenklatura 20 fois. L'armée et le KGB, sont, et cela est à noter, très peu cités. Des groupes sociaux, comme l'Eglise, les jeunes, les travailleurs collectifs, le mouvement paysan et les syndicats sont perçus comme ayant peu d'influence. On serait tenté de conclure que l'intérêt manifesté par de tels groupes est peu important et que la mobilisation de masse est très limitée. Cela est tout aussi vrai avant et après le coup d'Etat de 1992.

Il faut préciser que l'étude réputationnelle n'est qu'un moyen parmi d'autres pour étudier l'élite, les intérêts, les forces et les personnes impliqués dans le changement politique, et qu'elle doit donc être complétée par d'autres méthodes. Mais dans une période de changements intenses, c'est un des moyens d'information les plus intéressants pour identifier et définir les personnes et les institutions qui constituent et influencent le pouvoir politique.

Cette analyse confirme de nombreux aspects généraux de l'orientation politique du mouvement de réforme sous Gorbachev. L'influence varie avec le type de décision : les intérêts régionaux semblent plus décisifs dans l'élaboration du Traité de l'union, et les conseillers économiques plus influents dans l'introduction des principes de l'économie marché. Les personnes et institutions politiques ayant le plus d'influence semblent être des membres du Parti communiste de l'Union soviétique et de l'appareil. Eltsine était le seul leader n'appartenant pas à l'establishment à qui l'on attribuait un rôle important en tant que chef de la contre-élite. Gorbachev était confronté à d'autres groupes et institutions auxquels il réagissait en construisant sa politique. Les forces dans les républiques et les régions ainsi que le mouvement démocratique étaient de loin les plus importants, leurs influences se répartissaient selon des sphères d'intérêt: les démocrates soutenaient les réformes politiques et économiques, tandis que les groupes d'intérêts dans les républiques étaient surtout préoccupés par la redistribution du pouvoir entre le centre et les régions. Les forces extérieures, à savoir les chefs d'Etat étrangers, étaient censées avoir eu la plus grande influence sur la politique étrangère, sous Gorbachev. Il est certain que les politiques de transition ne peuvent être comprises indépendamment du système politique international ${ }^{19}$.

L'analyse réputationnelle du leadership sous Eltsine a montré que la structure et la composition de l'élite, ont changé de façon significative. Or, de tels changements systémiques ont sans nul doute contribué à une moindre intégration de l'élite politique. En effet, le pouvoir politique est caractérisé par un leadership fragmenté, et cela peut certainement contribué à une instabilité du régime. Les décisions portant sur des aménagements constitutionnels, l'économie ou les relations avec d'autres Etats de l'ancienne Union Soviétique, sont perçues comme étant influencées par différentes constellations d'individus et de forces politiques. Trois types de groupes d'intérêts apparaissent comme dominants: premièrement, la bourgeoisie et les forces démocratiques qui souhaitent un système politique fondé sur l'économie de marché, une société civile et le pluralisme, bien que des factions au sein de ce groupe donnent des priorités différentes à ces valeurs. Deuxièmement un groupe de forces nationales et de "patriotes", mettant l'accent sur la solidarité autour des valeurs nationales et 
traditionnelles. Troisièmement, les éléments de l'administration gouvernementale qui peuvent inclure des intérêts liés à l'ancien ordre économique et politique. Les considérations idéologiques sortent $\mathrm{du}$ cadre de cette étude, mais on pourrait remarquer que ces intérêts nourrissent des préférences différentes pour le type de système politique qu'ils cherchent à promouvoir. Nos interlocuteurs nous ont rappelé que la bourgeoisie naissante est présente dans le premier groupe tandis que l'idéologie administrative, traditionnelle (dont celle du système communiste) et nationale est le propre des deux autres. On pourrait penser que le troisième groupe - constitué des personnes ayant une position élevée dans l'appareil économique de l'Etat - occupe une position contradictoire. En effet, ils pourraient être confrontés de par les effets mêmes des réformes à une situation ambivalente. D'un côté, leur légitimité de par leur appartenance à la "nomenklatura" pourrait être mise en question, mais de l'autre, les privatisations notamment à travers la gestion de la mise vente des entreprises, peuvent être des occasions rêvées pour ces derniers de légitimer non seulement leur pouvoir économique mais également leur droit à la propriété.

Les réponses indiquent également quels sont les groupes et les forces qui n'ont pas ou peu d'influence. Les partis politiques (à l'exception peut-être de la Russie démocratique vers la fin de la période de Gorbachev) ne rassemblent autour d'eux que peu d'intérêts, et sont perçus comme ayant une influence minime. Le système de parti est demeuré sous une forme embryonnaire: des partis auto-proclamés très peu structurés et de petites associations d'individus sans programme véritablement défini, ni de véritables membres. Il est clair que le rôle des ouvriers, des paysans, et des syndicats est minime ; et que celui des anciens piliers de l'ordre politique - en particulier le KGB et l'armée semble négligeable. Ce manque d'influence peut être interprété de deux façons: un manque de mobilisation collective massive et donc l'absence de menace d'insurrection d'une contre l'élite politique; un manque d'intégration et de participation au régime, ce qui pourrait laisser présager un changement de régime plus significatif. Les forces armées ont été politiquement astucieuses. L'absence de rôle politique effectif au début de la période de transition indique qu'elles étaient sous le contrôle des civils. En même temps, elles ont décidé avec discrétion quand il fallait, et quand il ne fallait pas, intervenir. Mollement en août 1991, mais de façon décisive en octobre 1993. Ainsi, elles ont renforcé leur position d'arbitre indépendant entre les forces politiques.

En étudiant les forces sociales, on ne peut que conclure que les agents principaux d'un changement politique selon la théorie marxiste - les forces économiques nouvelles et la classe ouvrière - ont joué un rôle mineur dans la conduite du mouvement de réforme durant la dernière période. Si cela a été une révolution, elle a été politique, menée par le sommet et encouragée par deux types de contre-élites internes, les nationalistes et les démocrates, et surtout par les leaders conservateurs des nations occidentales les plus puissantes. L'intelligentsia a joué un rôle important en développant l'idéologie d'une réforme alternative sous Gorbachev. Sous Eltsine, son rôle a diminué. Elle a perdu son pouvoir d'initiative et d'influence.

De plus, nos interlocuteurs avaient conscience que la bourgeoisie était une force politique naissante dans le régime post-communiste. Dans le cas de la transition en Russie, les classes sociales ne semblent pas avoir appeler, via l'élite politique, à l'instauration d'un processus réforme. Les élites polotiques ont conduit le processus de changement et ont tenter de créer une structure de classe sur laquelle son futur pourrait reposer. La forme de la privatisation pourrait avoir une importance primordiale pour la stabilité future de la classe capitaliste. Il semble probable que 
l'ancienne nomenklatura (en particulier les titulaires des postes administratifs et exécutifs des ministères économiques) bénéficiera de façon tangible des possibilités de rachat des parts des biens propriétés de l'Etat et de ce fait légitimera ainsi sa position de classe aussi bien en termes de propriété privé que de possesseur d'actifs.

Cette étude veut indiquer et rappeler que l'on a assisté à une modification de la structure de l'élite - et non point seulement des individus qui la constituait. Ceci suppose qu'au début de la période de transition, les sources du conflit politique et les dynamiques du changement social résident non dans la mobilisations des masses mais les contradictions des objectifs et des intérêts de l'élite.

Les études des élites dans le processus de transition ont des implications importantes pour l'élaboration de la politique, car la stabilité du régime est fondée sur l'unité des élites $^{20}$. Le manque de consensus entre les élites nationales produit des régimes instables alternant entre autoritarisme et démocratie formelle. Dans la Russie d'aujourd'hui, les forces politiques en faveur de l'économie de marché, le pluralisme politique et la démocratie - dans le sens d'une compétition des partis politiques et des règlements négociés - ne bénéficient que d'un faible soutien. Pour accroître les chances de stabilité politique, l'exécutif politique d'Eltsine doit former une alliance avec l'un des autres groupes - les nationalistes ou l'élite administrative. Or, sans un pacte ou compromis, la désunion de l'élite s'intensifiera et accentuera la défaillance du gouvernement ainsi que l'impuissance du régime.

Le soutien politique est aussi fondé sur les intérêts de classe. Engels a écrit, en examinant les bases de la révolution: "Toute révolution...a été faite pour protéger un type de propriété contre un autre type de propriété. Elle ne peut en protéger un sans en violer un autre" ${ }^{12}$. Dans la structure de classe, le contrôle sur les biens est le fondement du clivage entre les acteurs principaux. A la fin de son "règne» Gorbachev, pour assurer le changement interne et l'acceptation par la communauté internationale, a adopté le libéralisme, le marché et la société civile. Mais, il dépendait de la classe traditionnelle base du socialisme d'Etat: la bureaucratie étatique et la direction des industries. Il occupait donc une position de classe contradictoire.

Le mouvement de réformes et le gouvernement d'Eltsine s'engagent de plus en plus aux côtés du marché et de la privatisation, et font appel aux intérêts bourgeois - ceux liés à la production et ceux liés à consommation. Mais la bourgeoisie est peu nombreuse et ne dipose que de peu de pouvoir social et économique. Ici, la privatisation présente une occasion de créer une classe, non point issue de la population dans son ensemble, mais en favorisant en particulier la nomenklatura économique traditionnelle, en lui donnant l'occasion de légitimer son pouvoir politique à travers la propriété des moyens de production .

Enfin, un pacte entre le leadership d'Eltsine et chacun des deux groupes mentionnés cidessus va affaiblir plusieurs éléments du mouvement de réformes dans sa volonté d'introduire l'économie de marché, la société civile et le pluralisme démocratique. Un marché sans contraintes peut conduire au chômage et à la pauvreté généralisée. La fragmentation de l'élite à laquelle on a fait allusion précédemment devraient inciter les gouvernements occidentaux à être extrêmement prudents en soutenant une force ou un leader plutôt qu'une autre ou un autre, dans l'espoir pieux qu'ils vont "introduire la démocratie et renforcer les réformes". Une telle politique augmenterait probablement plus qu'elle ne les diminuerait, les causes de l'instabilité, ce qui compromettrait certainement les réformes. Dans la Russie contemporaine, un mouvement rapide de 
l'économie centralisée vers la formation d'une économie basée sur le marché et la bourgeoisie, pourrait renforcer les conditions d'une désunion sociale.

Les gouvernements occidentaux pourraient ne pas avoir le choix entre d'un côté "la démocratie et les réformes" et de l'autre un gouvernement autoritaire, mais bien plutôt, entre un gouvernement autoritaire appuyant le marché et la bourgeoisie naissante, d'une part, et l'établissement d'une élite qui assurerait la stabilité du régime en limitant le développement du système démocratique de pluralisme politique à l'occidentale, d'autre part. Ni l'un ni l'autre n'entraînerait la stabilité fondée sur l'instauration de l'élite et un consensus démocratique - système qui a existé pendant des siècles dans des pays comme les Etats-Unis et l'Angleterre. La structure de leur régime font de ces pays des exceptions et il serait utopique de s'attendre à ce que les sociétés en transition de l'Europe de l'Est et de la Russie les rejoignent sans une longue période d'ajustement amer entre les élites. L'absence de leaders significatifs pour les forces de masse majoritaires (la classe ouvrière en particulier) avant octobre 1992 a empêché une mobilisation contre la nouvelle élite d'Eltsine, mais a aussi minimisé la participation à l'intérieur de celle-ci. Or, l'augmentation du mécontentement des masses pourrait avoir des conséquences incontrôlées et incontrolables. La politique devrait soutenir l'articulation des intérêts et la formation des coalitions, et plaider pour un compromis entre ces élites sur lequel la stabilité du régime, et éventuellement la démocratie, pourrait reposer.

\section{NOTES}

1. Je remercie l'ESRC anglais, qui a financé cette étude, dans la perspective d'un rapprochement Est-ouest. Je remercie aussi le Centre de recherches russes de l'Université de Harvard qui a facilité la rédaction de ce texte.

2. Dans l'étude des positions, on considère les personnes qui occupent des positions importantes. En utilisant la méthode de la réputation, on demande qui a du pouvoir et de l'influence, à ceux qui ont quelque autorité en la matière. On peut encore employer deux autres méthodes pour analyser le pouvoir politique : celle décisionnelle - fondée sur l'étude des décisions - et la méthode répartitionnelle, où on prend en considération les effets des décisions (qui en tire des bénéfices).

3. Depuis janvier 1993 le journal Nezavisimaia, a publié tous les mois un palmarès de cent hommes politiques basé sur une estimation faite à partir d'une liste de nom effectuée par cinquante experts appartenant aux médias et aux centres de recherche politique. Ces résultats ne sont pas strictement comparables avec cette recherche, puisqu'il n'a pas été demandé aux personnes interrogées de spécifier sur quelles questions les hommes politiques cités ont de l'influence.

4. 29 autres spécialistes ont été contactés mais ils n 'ont pas pu ou n'ont pas souhaité répondre. Deux questionnaires demeuraient incomplets, on a donc dû interroger deux autres nouvelles personnes .

5. "Qui, selon vous, a eu des influences décisives sur Gorbachev dans la formation du Traité de l'Union?" 
6. "Les premiers coopératives ont été mis sur pied sous Gorbachev. Qui, selon vous, a le plus influencé Gorbachev dans cette décision ? Donnez cinq noms."

7. "Sous Gorbachev, l'Union Soviétique a retiré ses forces armées de l'Europe de l'Est.

Qui, selon vous, a joué le rôle le plus important dans la prise de cette décision ? Donnez cinq noms."

8. "Quelles forces socio-économiques ont eu de l'influence dans la prise de cette décision (la formation du Traité de l'union) ? Donnez cinq noms."

9. "Quelles forces socio-économiques ont soutenu les coopératives ? En mentionnez cinq."

10. "Quelles forces socio-économiqes ont influencé cette prise de décision (de retirer les forces armées de l'Europe de l'Est) ? Donnez cinq réponses."

11. Question 9 : "Sous Eltsine le Parti communiste a été interdit. Qui, selon vous, a eu le plus d'influerce dans la prise de cette décision ? Donnez cinq noms."

12. Question 11 : "Sous Eltsine, l'URSS s'est dissoute et des Etats indépendants ont été formés. Qui, selon vous, a eu le plus d'influence dans cette décision ? Donnez cinq noms."

13. Question 13 : "Selon vous, qui soutient le plus les réformes de l'économie de marché ? Donnez cinq noms."

14. Question 7 : "Qui, (à part Eltsine) croyez vous être les cinq personnes les plus influentes ?"

15. "Sous Eltsine, on a interdit l'existence au Parti communiste. Quelles forces socioéconomiques ont eu de l'influence dans la prise de cette décision? Donnez jusqu'à cinq noms."

16. "Sous Eltsine, l'URSS a été dissoute, entraînant la formation de plusieurs Etats indépendants. Quelles forces socio-économiques ont influencé cette décision? Donnez jusqu'à cinq noms."

17. "Quels groupes socio-politiques ont soutenu l'extension des réformes du marché ?

18. "Quelles sont les cinq forces socio-politiques ayant le plus d'influence en Russie d'aujourd'hui ?

19. On doit à Peter Gourevitch à la fin des années 70 l'attention portée au fait que les forces exogènes influence la politique intérieure. Voir "The Second Image Reversed : The International sources of Domestic Politics", International Organisation, Autumn 1978, pp. 881-912

20. Cf. J. Higley, M. G. Burton, "The Elite Variable in Democratic Transitions and Breakdown", American Sociological Review, 1989, vol. 54, pp. 17-32

21. F. Engels, "The Origin of the Family, Private Poperty and the State", Selected Works, Moscow, 1951, p. 244.

\section{INDEX}

Index géographique : Russie

Mots-clés : professionels de la politique, crises politiques

Index chronologique : post-guerre froide 
AUTEUR

DAVID LANE

Reader in sociology à l'Université de Cambridge et Fellow of Emmanuel College. 